\title{
JTLU special issue editorial: Bicycling in changing urban regions
}

\author{
Kevin J. Krizek \\ University of Colorado Boulder \\ Radboud University \\ krizek@colorado.edu
}

\section{Karel Martens}

Radboud University

Technion-Israel Institute of Technology

kmartens@technion.ac.il

\author{
Fariya Sharmeen \\ Radboud University \\ Delft University of Technology \\ F.Sharmeen@fm.ru.nl
}

\section{$1 \quad$ The rise in research on bicycling}

As communities around the globe contemplate the future of their transport portfolio, bicycling's role has increasingly cropped up as a key discussion point. Up until a few years ago, bicycling's value was largely fueled by a loyal advocacy base. Its potential was littered with unsupported claims and bicycling struggled to obtain legitimate status, even as, or precisely because of, its status as a "fringe mode."

This context has recently changed. Concomitant with—or perhaps prompted by—a rise in (public and policy attention for) bicycling, there has been a rise in research specifically on bicycling. In just a few years, bicycling's stock has risen to be a mode that is commanding attention in cities of all sizes. Furthermore, its role and value are informed by a burgeoning evidence base, increasingly in the form of peer-reviewed work. This evidence base allows, among other things, a more reflective appreciation for bicycling's position in transport systems and for bicycling to be better understood in different geographical contexts.

A demonstration of such growth in attention can be seen via the Transportation Research Board (TRB) in the United States, which hosts a call for bicycling research as part of its annual conference. TRB's bicycling committee has reported a blistering increase in submissions over the past five years. Last year (2018) alone, it reviewed 141 submissions, up from 78 submissions in 2013; over the fiveyear period, this TRB committee reviewed 644 papers, covering research from an increasing number of countries. TRB is not the only venue that has seen a rapid uptick in activity. Furthermore, European platforms such as Cycling for Society and Scientists for Cycling have also witnessed an increase in both the quantity and quality of cycling research. A spirited dimension to these research initiatives is prizing

Copyright 2018 Kevin J. Krizek, Fariya Sharmeen \& Karel Martens

http://dx.doi.org/10.5198/jtlu.2018.1459

ISSN: 1938-7849 | Licensed under the Creative Commons Attribution - Noncommercial License 4.0

The Journal of Transport and Land Use is the official journal of the World Society for Transport and Land Use (WSTLUR) and is published and sponsored by the University of Minnesota Center for Transportation Studies. 
the need to furnish action-oriented research. This work with quick turnaround to help allow cities better realize what "works" and what doesn't as they gradually evolve towards sustainable mobility. ${ }^{1}$ Practitioners are increasingly and rightfully asking, "what does the evidence-base say?" This generates pressure on researchers to: (a) define the nature of the evidence base, its strengths and weaknesses, (b) translate such findings into practice and (c) do so quickly amidst the rapidly transforming industry of transport.

\section{Growing pains of cycling research}

Within this period of rapid growth, expected growing pains have been experienced. Significant as part of this process remain key challenges bicycling research; we draw attention to three of them, below.

1. Bicycling research reaches far beyond transport dimensions. While bicycling is principally considered a transport mode, there remain valuable perspectives from anthropology, policy, economics, health and others which can be important to bring to bear. Owing to "tunnel" vision that is common among academic disciplines, it is difficult to translate perspectives or knowledge between disciplines.

2. Methods and approaches are disparate. Ensuring consistent quality for the research is difficult, owing in large part to the standards of different disciplines. For example, while a traffic engineer and an anthropologist may be independently studying the same roadway intersection - using radically different methods - they may still be asked to review each other's work because of its bicycling orientated nature. This may lead to conflating standards or perception of perceived quality. Recognizing that this issue could theoretically be alleviated by directing reviews only to individuals who are both within a topic area and the discipline, that pool of reviewers within the field of bicycling is only recently starting to grow.

3. Advocacy sentiments often fuel the research - and the findings. In eyes of many transport researchers, cycling has fallen victim to being heavily influenced by a strong advocacy base. In other words, many cycling researchers "have the answer first" and look for the evidence to support it. In contrast, (arguably) a "proper" researcher is ready to "expect the unexpected" and then help build a supposedly "value neutral" evidence base given certain criteria.

\section{Velo-city 2017}

These three perspectives on the state of bicycling research provided a valuable backdrop for a major cycling event that was held in June 2017 in the Netherlands: Velo-city 2017, the largest gathering of bicycle-related professionals in the world. Over 2,000 participants were jointly hosted by the Province of Gelderland, the cities of Arnhem and Nijmegen, and Radboud University for a multi-day conference. Leveraging this conference and the Scientists for Cycling network, a one-day symposium ${ }^{2}$ was held prior to this event, to specifically discuss cycling research. It gathered 160 individuals, including university professors, doctoral students, student researchers, cycling professionals and others to "move the needle" with respect to "what is known" about bicycling focused research, from all walks of research life, all disciplines and all corners of the map.

No single outlet can adequately capture the knowledge that was shared. The products from this single day event were many. The full program included around 80 presentations. Three keynote presentations provided an inspiring starting point. Caspar Chorus (Professor of Choice Behavior Modeling

${ }^{1}$ For an overview access the archives of Cycling and Society conferences, including media documentations and blog posts, here http://www.cyclingandsociety.org/archive/

${ }^{2}$ Held in Nijmegen, the Netherlands on 12 June, 2017, https:/www.velo-city2017.com/extracurricular/scientific-colloquium/ 
from Delft University of Technology) kicked things off by sharing polemical perspectives about bicycling's role amidst conventional theories of travel behavior. He reviewed work in psychology suggesting that in the domain of morality, decision-making tends to be driven by emotions, heuristics, and an unwillingness to compromise. Based on this insight, he argued for a more dispassionate approach to policy-making, based on rigorous scientific studies into the causal determinants and effects of cycling behavior. Maria Hopman (Professor of Integrative Physiology, Radboud University and Radboudumc) alerted the congress to the valuable public health benefits of cycling. Leveraging the concept of "vitality" of individuals as they age from conception to decease, she unraveled the mechanisms that explain the relationship between inactivity and the development of chronic diseases as well. She concluded by highlighting the viable role that everyday cycling can provide to further exercise and health. Arnoud Lagendijk (Professor of Economic Geography, Radboud University) highlighted the value of practices and institutional configurations. He argued formal approaches of bicycling knowledge creation and application only serve to verify general insights of little practical use. Understanding and advocating bicycling warrants knowledge of how cycling evolves as part of life trajectories, social practices, and entrepreneurial initiatives.

These keynotes were supplemented with around 80 contributions - all peer reviewed-and five focused panel discussions. This was the first-ever gathering of cycling researchers of such magnitude and with such a rigorous peer review process. This special issue of JTLU captures six of the full papers with a clear relevance for transport and land use, as well as a viewpoint written by a science journalist specialized in the field of transportation who attended the event. Such outside perspective is a new approach that adds value to the overall debate, we believe. Other work presented at the conference has been published in other journals.

\section{Informing the nature of bicycling research: Where could we go from here?}

As the individuals primarily responsible for administering the one-day research symposium, we learned a lot. Based on the process leading up to the symposium and also informed by the presentations and hallway conversations during the congress, we share the following perspectives.

\subsection{Having research keep pace with changing needs}

Owing to technological developments and societal transformations, useful research is having difficulty keeping pace with transport innovations. In other words, the world is changing more quickly than bicycling research is able to suitably address. Bicycling is not alone in this respect, but bicycle technology in the form of dockless bicycle sharing, peer-to-peer bicycle sharing, e-bikes and cargo bikes provide rich avenues for new lines of investigation.

Fortunately, the stock of world-wide researchers who are now studying cycling is providing some relief in this regard. For instance, there are already several papers on dockless bicycle sharing, while the product has been only around for a few years (Fishman, 2016; Parkes, Marsden, Shaheen, \& Cohen, 2013; Shen, Zhang, \& Zhao, 2018). Cargo bikes are still a fringe mode, both for businesses and for private users, yet there is already a small community of researchers studying this particular phenomenon (Riggs, 2016; Masterson, 2017). Bicycling research is slowly becoming a "normal" domain of research; the academic community is, by now, sufficiently large to cover a wide range of topics, with substantial numbers of researchers studying "mainstream" bicycling topics and a range of "lonely riders" covering "niches" and emerging trends that could not be addressed when the bicycling research community was much smaller in size. 
Yet, while the increase in bicycling research is certainly a positive development, it also reflects a more fundamental and problematic trait of transport research: its focus on transport modes and technologies rather than people. The boom in bicycle research is a case in point. However, this is overshadowed by the exponential increase of studies into autonomous vehicles. The latter especially seems to follow the path well-known from the early decades of transport research, with its focus on the best ways to optimize and accommodate the technology of the future. Formerly, it was the regular, privately owned, car. Now, it is the electrical, possibly shared, self-driving car. Some bicycling research appears to be following the same path, focusing on how to optimize the use and experience of bicycling through better design of infrastructure and facilities.

While bicycling has a multitude of benefits, for both user and society, and much can be gained by a higher increase in bicycle shares, there remains a risk that the machine is overshadowing the human. Research centered around a single transport mode tends to ignore that virtually every transport mode is exclusionary in nature, perfectly matching the needs and desires of some but not of others. Like all other modes, the bicycle is excellent for some people and for some trips purposes, but not for others. Cycling research, and policies based on cycling research, may thus unintentionally contribute to what is now known as transport poverty and transport-related social exclusion. This phenomenon is a direct result of car-centered transport policies, supported by a mode-oriented transport research agenda. Bicycling researchers could benefit from being aware of this risk and avoid becoming part of the problem of the future, just like much car-oriented research is still part of the problem rather than the solution for the challenging problems in the transport domain.

\subsection{Contexts vary and external validity suffers}

Findings from cycling research in one context might have little value elsewhere. While this is always the case, the fact that bicycling can be particularly woven into the culture of a place (e.g., like food) suggests that findings within a particular discipline (i.e., engineering) would be best relevant within the context in which the research was conducted. A lack of attention for context is not specific to bicycling research. Much transport research has historically paid little attention to the role of context and culture. This is, for instance, evident in studies into value of time, (transport mode) choice experiments, car-following, etc. Perhaps telling is the fact that even the Netherlands employed transport demand models that did not include cycling as a mode of transport until the late 1990s, even though the bicycle share was substantially higher than public transport share on many links.

In many transport research domains, only the second wave of studies became more sensitive to the fact that travel or mobility is an inevitably social and societal phenomenon and thus strongly interwoven with local context, including climate, culture, everyday practices, and policies in a range of domains. A well-known example of such embeddedness is the use of the bicycle among Dutch youth. The "popularity" of cycling among Dutch high schools is as much related to the institutional structure of education as to the existence of high-quality bicycle infrastructures. Indeed, it was the very organization of Dutch education that initially led to high levels of cycling among youth, which then triggered the outrage about poor traffic safety and the call for an improved bicycle infrastructure in the early 1970s. Furthermore, the current high bicycle use is sustaining itself not merely because of the bicycle infrastructure, but as much because of the social practices that emerged around cycling and made high school cycling a selfsustaining practice. Merely building safe bicycle lanes to high schools will not produce these same patterns in other countries. We would argue that cycling researchers have gone much quicker through the learning curve about the relevance of context than many other research strands in transport. The neglect in much of the research on autonomous vehicles for cultural and spatial context is a case in point. 


\section{Overview of the six contributions in this issue of JTLU}

The first paper (Furth et al., 2018) provides an example of the increased emphasis of planning and practice-oriented research. The authors analyze alternate low-stress bicycle route alignments in northern Delaware (US). Responding to the need of local authorities to evaluate and compare alternatives of infrastructure investments, they highlight the need of having comparative route alignments avoiding high-traffic high-stress links while minimizing detours. Their proposed propensity model offers a tool to measure network connectivity, thereby improving accessibility for both work and non-work trips.

The research from Yuan et al. (2018) focuses on the user in their cyclists' interaction behavior model. They narrate the interactions of cyclists in a controlled laboratory experiment as an alternative approach to collect traffic data. The model dissects individual maneuvering trajectories to avoid collision with other users. The results also point to subtle gender differences in cycling behavior and the model results contribute to informing safe cycling infrastructure design.

Collisions were explored in Williams et al. (2018) as influenced by built environment characteristics. This work investigates bicycle-motor collisions using a case control approach in New Zealand by studying how different features of road design affect bicycle crash occurrences, with an aim to identify high-risk areas and built environment features. They use network models to identify routes and use actual crash data of Christchurch to compute logistic regression models for likelihood analysis. Intersections and high arterial roads were found to be associated with crash risks as opposed to local residential streets.

Similar findings were reported in Barajas (2018), where safety and its relation to built environment characteristics takes the central focus, with a particular focus on disadvantaged neighborhoods. They explore bicycle crashes in the San Francisco Bay area to identify potential differences in bicycle crash occurrences in ethnic neighborhoods. The study appeals to the need for city planners to take neighborhood vulnerabilities and sociodemographic disparities into account.

Focusing on more behavioral analysis, Bernardi et al. (2018) evaluate individuals' route choice using smartphone data. They employ discrete choice models referring to an array of cycling use, from frequent cyclists, systematic, and repeated trips to non-leisure travels, linking to route choices. Remarkably, shortest routes were not always found to be the chosen option. Particularly for discretionary trips, personalized routes seem to be preferred by Dutch cyclists. This research highlights interpersonal as well as trip-related attributes in the choice of cycling routes.

In the context of cycling innovations to enable seamless longer commutes, van den Berg et al. (2018) explored behavioral choice on solar bicycles. This is a special category of electric pedal assisted bicycle with solar panels in the front wheels, thereby enabling independent charging through sunlight. The study conducts a stated preference survey data to model mode-choice as a function of sociodemographic latent classes and trip-related attributes. Contrary to popular belief, their findings suggest that women and retired individuals are among the main users of this new technology.

In sum, a solid research base on bicycling is critical to support larger initiatives as communities reinvent their transport portfolio. We trust you find value in how the articles and perspectives in this special issue of JTLU further reinforce this aim. 


\section{References}

Barajas, J. M. (2018). Not all crashes are created equal: Associations between the built environment and disparities in bicycle collisions. Journal of Transport and Land Use, 11(1), 865-882. doi: 10.5198/ jtlu.2018.1145

Bernardi, S., Geurs, K., \& La Paix Puello, L. (2018). Modelling route choice of Dutch cyclists using smartphone data. Journal of Transport and Land Use, 11(1), 883-900. doi: 10.5198/jtlu.2018.1143

Furth, P. G., Moser, P., \& Putta, T.V.V.K. (2018). Measuring low-stress connectivity in terms of bikeaccessible jobs and potential bike-to-work trips: A case study evaluating alternative bike route alignments in northern Delaware. Journal of Transport and Land Use, 11(1), 815-831. doi: 10.5198/ jtlu.2018.1159

Fishman, E. (2016). Bikeshare: A review of recent literature. Transport Reviews, 36(1), 92-113.

Masterson, A. (2017). Sustainable urban transportation: Examining cargo bike use in Seattle (Dissertation). University of Washington, Seattle, WA.

Parkes, S. D., Marsden, G., Shaheen, S. A., \& Cohen, A. P. (2013). Understanding the diffusion of public bikesharing systems: Evidence from Europe and North America. Journal of Transport Geography, $31,94-103$.

Riggs, W. (2016). Cargo bikes as a growth area for bicycle vs. auto trips: Exploring the potential for mode substitution behavior. Transportation Research Part F: Traffic Psychology and Behavior, 43, 48-55.

Shen, Y., Zhang, X., \& Zhao, J. (2018). Understanding the usage of dockless bike sharing in Singapore. International Journal of Sustainable Transportation, 12(9), 686-700.

van den Berg, P., Vinken, S., Geurs, K., \& Arentze, T. (2018). Stated choice model of transport modes including solar bike. Journal of Transport and Land Use, 11(1), 901-919. doi: 10.5198/jtlu.2018.1149

Williams, T., Page, S., \& Doscher, C. (2018). Spatial characteristics of bicycle-motor vehicle crashes in Christchurch, New Zealand: A case-control approach. Journal of Transport and Land Use, 11(1), 849-864. doi: 10.5198/jtlu.2018.1147

Yuan, Y., Goñi-Ros, B., Daamen, W., \& Hoogendoorn, S. (2018). Investigating cyclist interaction behav-ior through a controlled laboratory experiment. Journal of Transport and Land Use, 11(1), 833-847. doi: 10.5198/jtlu.2018.1155 